\title{
Correction to: Sex differences in the association between plasma copeptin and incident type 2 diabetes: the Prevention of Renal and Vascular Endstage Disease (PREVEND) study
}

\author{
A. Abbasi ${ }^{1,2} \cdot$ E. Corpeleijn ${ }^{1} \cdot$ E. Meijer ${ }^{2} \cdot$ D. Postmus ${ }^{1} \cdot$ R. T. Gansevoort ${ }^{2} \cdot$ R. O. B. Gans ${ }^{2} \cdot$ J. Struck ${ }^{3} \cdot$ H. L. Hillege ${ }^{1}$. $^{2}$ \\ R. P. Stolk ${ }^{1}$ - G. Navis ${ }^{2}$ - S. J. L. Bakker ${ }^{2}$
}

Published online: 12 June 2019

(C) Springer-Verlag GmbH Germany, part of Springer Nature 2019

\section{Correction to: Diabetologia}

https://doi.org/10.1007/s00125-012-2545-x

The values given for copeptin levels in men in quartiles 1 and

2 (Table 1) were incorrect, and should have read:

Quartile 1: $3.0(2.3-3.5)$

Quartile 2: 5.1 (4.6-5.6)

The total hs-CRP value in men should have read $1.20(0.55-$ 2.64).

The corrected table is reproduced here.

The online version of the original article can be found at https://doi.org/ $10.1007 / \mathrm{s} 00125-012-2545-\mathrm{x}$

A. Abbasi

a.abbasi@umcg.nl

1 Department of Epidemiology, University of Groningen, University Medical Center Groningen, Hanzeplein 1, P.O. Box 30.001, 9700 RB Groningen, the Netherlands

2 Department of Internal Medicine, University of Groningen, University Medical Center Groningen, Groningen, the Netherlands

3 Department of Research, BRAHMS GmbH/Thermo Fisher Scientific, Hennigsdorf, Germany 
Table 1 Baseline clinical characteristics of participants in total and according to quartiles of plasma copeptin

\begin{tabular}{|c|c|c|c|c|c|c|}
\hline \multirow[t]{2}{*}{ Characteristic } & \multirow[t]{2}{*}{ Total } & \multicolumn{4}{|c|}{ Sex-specific quartiles } & \multirow[t]{2}{*}{$p$ value $^{\mathrm{a}}$} \\
\hline & & 1 & 2 & 3 & 4 & \\
\hline \multicolumn{7}{|l|}{ Women } \\
\hline No. of participants & 4063 & 1001 & 1025 & 1019 & 1018 & - \\
\hline Copeptin level (pg/ml) & $3.6(2.4-5.5)$ & $1.8(1.4-2.1)$ & $2.9(2.6-3.2)$ & $4.4(4.0-4.9)$ & $7.6(6.3-9.8)$ & - \\
\hline Age (years) & $47.7 \pm 12.2$ & $46.7 \pm 12.2$ & $46.8 \pm 11.8$ & $47.8 \pm 12.2$ & $49.6 \pm 12.3$ & $<0.001$ \\
\hline Family history of diabetes (\%) & $830(20.4)$ & $202(20.2)$ & $190(18.5)$ & $208(20.4)$ & $230(22.6)$ & 0.16 \\
\hline Current smoker $(\%)$ & $1374(33.8)$ & $275(27.5)$ & $313(30.5)$ & $366(35.9)$ & $420(41.3)$ & $<0.001$ \\
\hline Ever use alcohol (\%) & $2718(67.2)$ & $641(64.2)$ & $707(69.2)$ & $684(67.5)$ & $686(67.9)$ & 0.10 \\
\hline BMI $\left(\mathrm{kg} / \mathrm{m}^{2}\right)$ & $25.8 \pm 4.6$ & $25.4 \pm 4.1$ & $25.6 \pm 4.5$ & $26.0 \pm 4.7$ & $26.2 \pm 5.2$ & $<0.001$ \\
\hline Waist circumference (cm) & $82.9 \pm 12.4$ & $81.2 \pm 11.1$ & $82.5 \pm 12.3$ & $83.4 \pm 12.3$ & $84.3 \pm 13.6$ & $<0.001$ \\
\hline Systolic blood pressure (mmHg) & $119.4 \pm 19.5$ & $118.3 \pm 18.7$ & $118.4 \pm 18.6$ & $119.7 \pm 19.8$ & $121.1 \pm 20.9$ & 0.003 \\
\hline Diastolic blood pressure $(\mathrm{mmHg})$ & $68.7 \pm 9.0$ & $68.3 \pm 8.9$ & $68.5 \pm 8.8$ & $68.7 \pm 9.2$ & $69.4 \pm 9.0$ & 0.03 \\
\hline Hypertension (\%) & $959(23.6)$ & $222(22.2)$ & $216(21.1)$ & $235(23.1)$ & $286(28.1)$ & 0.001 \\
\hline Glucose (mmol/l) & $4.62 \pm 0.64$ & $4.61 \pm 0.60$ & $4.59 \pm 0.59$ & $4.61 \pm 0.62$ & $4.67 \pm 0.71$ & 0.04 \\
\hline Total cholesterol (mmol/l) & $5.60 \pm 1.14$ & $5.56 \pm 1.15$ & $5.51 \pm 1.12$ & $5.61 \pm 1.15$ & $5.71 \pm 1.15$ & 0.001 \\
\hline HDL-cholesterol (mmol/l) & $1.49 \pm 0.41$ & $1.49 \pm 0.41$ & $1.49 \pm 0.40$ & $1.47 \pm 0.39$ & $1.50 \pm 0.41$ & 0.44 \\
\hline Triacylglycerol (mmol/l) & $1.05(0.78-1.46)$ & $1.02(0.76-1.43)$ & $1.04(0.77-1.45)$ & $1.06(0.78-1.48)$ & $1.06(0.80-1.50)$ & 0.13 \\
\hline hs-CRP (mg/l) & $1.31(0.55-3.21)$ & $1.20(0.50-3.19)$ & $1.26(0.53-3.01)$ & $1.35(0.61-3.27)$ & $1.46(0.58-3.34)$ & 0.03 \\
\hline UAE (mg/24 h) & $10.4(6.8-20.6)$ & $7.3(5.5-11.7)$ & $8.2(5.7-13.1)$ & $8.3(5.8-13.7)$ & $9.8(6.3-17.7)$ & $<0.001$ \\
\hline \multicolumn{7}{|l|}{ Men } \\
\hline No. of participants & 3909 & 973 & 980 & 978 & 978 & - \\
\hline Copeptin level (pg/ml) & $6.2(4.0-9.4)$ & $3.0(2.3-3.5)$ & $5.1(4.6-5.6)$ & $7.6(6.9-8.5)$ & $12.5(10.5-15.5)$ & - \\
\hline Age (years) & $50.2 \pm 1.8$ & $49.3 \pm 12.6$ & $49.5 \pm 12.9$ & $49.9 \pm 12.6$ & $52.2 \pm 12.8$ & $<0.001$ \\
\hline Family history of diabetes (\%) & $753(19.3)$ & $187(19.2)$ & $169(17.2)$ & $206(21.1)$ & $191(19.5)$ & 0.20 \\
\hline Current smoker $(\%)$ & $1369(35.0)$ & $319(32.8)$ & $347(35.4)$ & $361(36.9)$ & $342(35.0)$ & 0.29 \\
\hline Ever use alcohol (\%) & $3210(82.5)$ & $794(81.8)$ & $821(84.3)$ & 798 (81.9) & 797 (81.9) & 0.40 \\
\hline BMI $\left(\mathrm{kg} / \mathrm{m}^{2}\right)$ & $26.2 \pm 3.6$ & $25.8 \pm 3.4$ & $26.1 \pm 3.5$ & $26.4 \pm 3.6$ & $26.5 \pm 3.8$ & $<0.001$ \\
\hline Waist circumference (cm) & $93.6 \pm 10.9$ & $92.6 \pm 10.4$ & $92.7 \pm 10.9$ & $94.0 \pm 10.8$ & $94.9 \pm 11.4$ & $<0.001$ \\
\hline Systolic blood pressure (mmHg) & $128.7 \pm 17.8$ & $126.5 \pm 17.3$ & $127.5 \pm 17.5$ & $129.9 \pm 17.4$ & $131.0 \pm 18.8$ & $<0.001$ \\
\hline Diastolic blood pressure $(\mathrm{mmHg})$ & $74.7 \pm 9.5$ & $73.2 \pm 9.1$ & $74.2 \pm 9.4$ & $75.2 \pm 9.3$ & $76.1 \pm 10.0$ & $<0.001$ \\
\hline Hypertension (\%) & $1278(32.7)$ & $273(28.1)$ & $317(32.3)$ & $316(32.3)$ & $372(38.0)$ & $<0.001$ \\
\hline Glucose (mmol/l) & $4.86 \pm 0.66$ & $4.82 \pm 0.66$ & $4.80 \pm 0.61$ & $4.86 \pm 0.65$ & $4.95 \pm 0.70$ & $<0.001$ \\
\hline Total cholesterol (mmol/l) & $5.68 \pm 1.10$ & $5.60 \pm 1.04$ & $5.64 \pm 1.10$ & $5.66 \pm 1.09$ & $5.81 \pm 1.17$ & $<0.001$ \\
\hline HDL-cholesterol (mmol/l) & $1.16 \pm 0.31$ & $1.17 \pm 0.31$ & $1.16 \pm 0.30$ & $1.15 \pm 0.31$ & $1.17 \pm 0.35$ & 0.19 \\
\hline Triacylglycerol (mmol/l) & $1.28(0.91-1.88)$ & $1.24(0.89-1.75)$ & $1.23(0.90-1.80)$ & $1.30(0.93-1.90)$ & $1.35(0.95-2.00)$ & $<0.001$ \\
\hline hs-CRP (mg/l) & $1.20(0.55-2.64)$ & $1.03(0.47-2.28)$ & $1.08(0.52-2.50)$ & $1.22(0.58-2.74)$ & $1.45(0.70-3.11)$ & $<0.001$ \\
\hline UAE (mg/24 h) & $8.3(5.8-13.9)$ & $9.3(6.4-17.2)$ & $9.7(6.6-19.3)$ & $10.7(7.1-20.0)$ & $11.9(7.4-27.9)$ & $<0.001$ \\
\hline
\end{tabular}

Data are given as mean \pm SD or median (IQRs) for continuous variables, tested using ANOVA or the Kruskal-Wallis test, and numbers (percentage) for categorical variables, tested using the $\chi^{2}$ test

${ }^{a}$ Univariate analyses were for comparison across sex-specific quartiles of plasma copeptin

Publisher's note Springer Nature remains neutral with regard to jurisdictional claims in published maps and institutional affiliations. 